\title{
Perspectivas sobre o Aprendizado na Óptica de Estudantes de Medicina: Análise do Impacto de Transição Curricular
}

\author{
Perspectives on Learning From the Viewpoint of \\ Medical Students: Curriculum Transition Impact \\ Analysis
}

Paulo Roberto Sotillo de Lima Filho ${ }^{I}$

Rossana Vanessa Dantas de Almeida Marques

\section{PALAVRAS-CHAVE}

- Educação Médica.

- Aprendizagem.

- Avaliação Educacional.

- Ensino; Currículo.
Com o avanço tecnológico e as modificações políticas e socioculturais ocorridos ao longo dos anos, as perspectivas educacionais vão se remodelando, com utilização de novas metodologias de ensino e ampliação do número de vagas. Com esses novos ambientes educacionais, surgem novos desafios e necessidade de adaptação. Entre os componentes que influenciam o processo de aprendizagem, procuramos, neste estudo, compreender a percepção dos discentes de um novo curso de graduação em Medicina quanto aos aspectos de infraestrutura, do corpo docente e tutoriais, do currículo informal, composto por grupos acadêmicos organizados pelos próprios alunos, e da organização didático-pedagógica. A pesquisa foi realizada por meio de questionário com 31 itens, com opções de resposta na escala do tipo Likert, envolvendo 208 alunos regularmente matriculados do segundo ao oitavo período. Estes foram divididos em dois grupos: um composto por alunos entre o quinto e o oitavo período (G2), que passaram por um processo de transição entre a metodologia mista, com aspectos tradicionais e algumas disciplinas ativas, para metodologia ativa; e outro por alunos do segundo ao quarto período (G1), que desde o primeiro período já utilizavam as metodologias ativas. Os alunos avaliaram como satisfatórios $41,9 \%$ dos itens questionados e como insatisfatórios 19,3\% dos itens. Os itens que mais foram considerados satisfatórios estavam relacionados a interdisciplinaridade, bibliografia recomendada, uso de metodologias ativas, integração com a comunidade, práticas de ensino, titulação docente e equipamentos didáticos. Os principais pontos de discordância entre os grupos estão relacionados aos aspectos didático-pedagógicos, como articulação teoria-prática, interdisciplinaridade, flexibilidade curricular, pertinência dos conteúdos, integração com a comunidade e atividades práticas. Questões relacionadas a número de docentes por alunos, práticas de ensino nos períodos mais avançados, componentes da biblioteca e apoio psicopedagógico foram os mais criticados pelos alunos. De forma geral, apesar das diversas dificuldades encontradas no contexto de um curso novo, houve mais pontos positivos que negativos na influência do aprendizado. Ainda assim, são necessárias algumas reestruturações para garantir a melhor experiência no processo ensino-aprendizagem. 


\section{KEY-WORDS}

- Education, Medical.

- Learning.

- Program Evaluation.

- Teaching; Curriculum

Recebido em: 16/8/18

Aceito em: 1/10/18

\begin{abstract}
Along with the technological advances and political and socio-cultural changes that have taken place over the years, educational perspectives have been reshaped, bringing new teaching methods and an expansion in the number of places. With these new educational environments, new challenges have arisen, and the need to adapt. Among the components that influence the learning process, this study seeks to understand the students' perceptions of a new undergraduate course in Medicine in terms of its infrastructure, faculty and tutorials, its informal curriculum, composed of academic groups organized by the students and its didactic and pedagogical organization. Our research involved a questionnaire with 31 items, with response options on a Likert scale, which was applied to 208 students enrolled in the second to eighth periods. We divided the students into two groups: one composed of students in the fifth to eighth periods (G2), who had transitioned from a mixed teaching methodology, with traditional aspects and some active disciplines, to an active methodology; and a second group composed of students in the second to the fourth periods (G1), who had begun using active methodologies right from the start of their course. The students evaluated $41.9 \%$ of the items questioned as satisfactory and $19.3 \%$ as unsatisfactory. The items that were deemed most satisfactory were related to interdisciplinary contents and approach, recommended bibliography, use of active methodologies, integration with the community, teaching practices, teacher qualification and didactic equipment. The main points of disagreement between the groups related to didactic and pedagogical aspects, such as the articulation of theory and practice, interdisciplinary contents, curricular flexibility, relevance of the contents, integration with the community and practical activities. The issues that received most criticism form the students were related to the teacher-to-student ratio, teaching practices in the more advanced periods, library components and psychological and pedagogical support. In general, we can see that despite the many difficulties encountered, in the context of a new course, there were more positives than negatives that influenced learning. Nevertheless, the program needs some restructuring to ensure the best experience in the teaching-learning process.
\end{abstract}

\section{INTRODUÇÃO}

As perspectivas sociais acerca dos papéis de professor e aluno vão se remodelando ao longo das gerações, com o surgimento de novas metodologias e abordagens pedagógicas distintas, que determinam novas formas de ensinar e de aprender. Nesse contexto educacional, o professor possui importante papel modulador, afetando o aprendizado por meio de seu conjunto de habilidades, onde a comunicação eficaz com os alunos é o principal componente ${ }^{1,2}$.

O ambiente de aprendizado ultrapassa a questão da transmissão de conteúdos teóricos, sendo um ambiente de valores e comportamentos que envolve troca de experiências e crescimento mútuo, influenciando o comportamento e o pensamento discentes ${ }^{3}$. Este tipo de interação é ainda mais importante na situação atual, em que os alunos podem obter informações em segundos; no entanto, apesar da importância do componente estrutural, o aprendizado também pode ser eficiente mesmo em ambientes desafiadores ${ }^{4}$.
No intuito de acompanhar essa transformação social, as instituições de ensino, especialmente os cursos de Medicina, têm adotado cada vez mais as metodologias ativas, que têm como princípio norteador a autonomia do estudante, numa visão de libertação educacional, onde o indivíduo deve aprender a buscar alternativas viáveis para a construção de seu próprio conhecimento ${ }^{5}$. Tais mudanças são evidenciadas nas Diretrizes Curriculares Nacionais do curso de graduação em Medicina, publicadas nos anos de $2001^{6}$ e $2014^{7}$, porém ainda há evidente limitação de direcionamento prático sobre como prover a melhor qualidade de ensino com uso dessas metodologias ${ }^{8}$.

Além disso, foi ampliado o número de vagas do Programa Nacional Mais Médicos, visando redistribuir a formação médica ao longo do território nacional ${ }^{9}$, existindo menor disponibilidade de recursos e preocupação com a qualidade de ensino, uma vez que os alunos já estejam matriculados. Assim, questões básicas como estrutura física adequada, são muitas vezes negligenciadas ${ }^{10}$. 
Tais processos ocorreram na Universidade Federal do Maranhão (UFMA), campus Imperatriz, com a criação, em 2014, do primeiro curso de graduação em Medicina no Sul do Maranhão. Inicialmente, foi adotada uma proposta metodológica mista - com aulas no método tradicional, associadas a disciplinas baseadas em metodologias ativas, como Aprendizagem Baseada em Problemas e Arco de Maguerez -, que foi substituída dois anos depois por metodologias ativas. Nos dois primeiros anos, o curso enfrentou diversas dificuldades, como atraso na entrega das instalações físicas e mudança da estrutura curricular para as turmas já matriculadas. Atualmente, o curso oferece 40 vagas semestrais e conta com 51 docentes, entre os quais 6 possuem doutorado, 16 possuem mestrado e 29 são especialistas.

O município de Imperatriz localiza-se no Oeste do Estado do Maranhão, com população estimada em 254.569 habitantes, densidade demográfica de 185,95 (hab $\left./ \mathrm{km}^{2}\right)$ e IDH de 0,731 , sendo considerada a segunda cidade mais populosa do estado e distante 637 quilômetros da capital São Luís ${ }^{11}$. A rede de saúde tem o papel de Centro de Referência em Saúde para os municípios que a circundam e até mesmo para os estados vizinhos, como Pará e Tocantins, e apresenta atualmente 398 estabelecimentos de saúde distribuídos no setor público (administração pública e entidades sem fins lucrativos; $n=100$ ) e privado (entidades empresariais e pessoa física; $n=298$ ), correspondendo a 7,46\% do Estado do Maranhão ${ }^{12}$, que incluem o Hospital Municipal de Imperatriz, o Hospital Municipal Infantil de Imperatriz, o Hospital Regional Materno Infantil, o Hospital Macrorregional, duas Unidades de Pronto Atendimento (UPA) e cinco Unidades Básicas de Saúde (UBS), que servem, por meio de convênios, como cenários de práticas para os estudantes do curso de Medicina da UFMA, campus Imperatriz.

Os discentes podem fornecer feedback valioso para a revisão e adequação das grades curriculares e do ambiente de aprendizagem, com novas percepções de suas experiências educacionais e áreas de preocupação, que devem ser melhoradas ${ }^{13}$. $\mathrm{O}$ processo de avaliação, no contexto institucional, leva à reflexão e, por consequência, à possibilidade de mudança da relação entre o processo e o produto das atividades educacionais ${ }^{14}$.

Nessa perspectiva, surge a seguinte questão: qual a percepção do acadêmico sobre as questões de infraestrutura, corpo docente e organização didática pedagógica? Diante desta questão, o presente estudo se propôs analisar a influência da infraestrutura, enquanto componente acadêmico, dos personagens envolvidos, como corpo docente e grupos acadêmicos, e da organização didático-pedagógica na percepção discente de construção do aprendizado do acadêmico do curso de Medicina.

\section{MÉTODOS}

A metodologia utilizada foi a analítica observacional transversal, com amostragem estratificada, composta por acadêmicos do curso de Medicina ( $n=208)$, alocados em dois grupos: G1 - acadêmicos matriculados do segundo ao quarto período que ingressaram após a mudança curricular, ou seja, que tiveram contato exclusivamente com metodologias ativas; G2 - acadêmicos matriculados do quinto ao oitavo período que tiveram contato ao menos durante um semestre com a metodologia mista.

Os dados foram coletados durante o segundo semestre letivo do ano de 2017 entre os estudantes universitários do curso de Medicina da Universidade Federal do Maranhão, campus Imperatriz. Foi aplicado um questionário individual, composto por questões que permitiam cinco níveis de respostas, conforme proposto pela escala de Likert: 1 - insatisfatório ou desfavorável; 2 - insuficiente ou pouco favorável; 3 - regular; 4 - satisfatório ou favorável; 5 - muito satisfatório ou muito favorável, o que possibilitou a análise quantitativa dos dados, compreendendo os fatores de forma isolada e integrada com relação aos aspectos investigados. As respostas mensuradas por essa escala foram agrupadas em conceitos insatisfatório (escores 1 e 2), regular (escore 3) e satisfatório (escores 4 e 5). Todos os participantes da pesquisa receberam e assinaram o Termo de Consentimento Livre e Esclarecido.

As questões abordaram aspectos curriculares, docentes / acadêmicos e estruturais e foram baseadas no Instrumento de Avaliação dos Cursos de Graduação do Instituto Nacional de Estudos e Pesquisas Educacionais Anísio Teixeira, do Ministério da Educação, publicado em abril de 2016, que subsidia as autorizações e reconhecimento dos cursos de graduação no Brasil $^{15}$. Adicionalmente, foram incluídas questões relacionadas à contribuição do currículo informal no aprendizado, a exemplo da participação em ligas acadêmicas.

O processamento dos dados foi realizado por meio do software estatístico Statistical Package for the Social Sciences (SPSS versão 22). A comparação entre os grupos foi realizada pelo método de correlação de Pearson, admitindo-se $\mathrm{p}<0,05$ como estatisticamente significativo e com intervalo de confiança de $95 \%$.

\section{RESULTADOS}

A média de idade dos discentes foi 23,09 $( \pm 4,31)$, com idade mínima de 17 anos e máxima de 41. Dos participantes, 48,1\% foram agrupados como G1, e 51,9\% como G2. Informações adicionais sobre a caracterização da amostra estão detalhadas na Tabela 1. 


\begin{tabular}{lcr}
\multicolumn{3}{c}{ TA BELA 1 } \\
& Caracterização da amostra \\
Variáveis & $\mathbf{n}$ & $\%$ total (\% G1/G2) \\
Gênero & & \\
$\quad$ Masculino & 121 & $58,2(52,5 / 47,5)$ \\
Feminino & 87 & $41,8(46,2 / 57,8)$
\end{tabular}

Possui alguma formação superior anterior?

$\begin{array}{lcc}\text { Sim } & 38 & 18,3(52,6 / 47,4) \\ \text { Não } & 170 & 81,7(51,8 / 48,2)\end{array}$

Participa ou participou de ligas acadêmicas?

$\begin{array}{lcc}\text { Sim } & 127 & 61,1(72,5 / 27,5) \\ \text { Não } & 81 & 38,9(19,7 / 81,3)\end{array}$

Com relação às respostas dos discentes, as porcentagens e comparações entre os grupos G1 e G2 foram agrupadas em quatro dimensões: organização didático-pedagógica; corpo docente e tutorial; estrutura física; contribuição do currículo informal no aprendizado (Tabela 2).

Das 31 questões utilizadas, 13 (41,9\%) obtiveram frequência superior a $50 \%$ na categoria satisfatório (níveis 4 ou 5 na escala Likert). Em contrapartida, apenas seis itens (19,3\%) foram classificados como insatisfatórios por mais de $50 \%$ dos participantes (níveis 1 ou 2 na escala).

Os maiores índices de satisfação estavam relacionados a interdisciplinaridade, bibliografia recomendada, uso de metodologias ativas, integração com a comunidade, atividades prá-

\begin{tabular}{|c|c|c|c|c|c|c|c|c|c|c|}
\hline \multirow{3}{*}{ Dimensões e questões avaliadas } & os G & $\begin{array}{r}\text { TABE } \\
\text { G2, ag } \\
\text { a física }\end{array}$ & $\begin{array}{l}\text { LA } 2 \\
\text { rupad } \\
\text { e cont } 1\end{array}$ & $\begin{array}{l}\text { segu } \\
\text { uiçã }\end{array}$ & $\begin{array}{l}0 \text { as } \\
\text { cur }\end{array}$ & dlo i & $\begin{array}{r}\text { orga } \\
\text { rma }\end{array}$ & $\begin{array}{l}\text { zação } \\
\text { apr }\end{array}$ & $\begin{array}{l}\text { dático- } \\
\text { izado }\end{array}$ & \multirow{3}{*}{$p$-valor } \\
\hline & \multicolumn{3}{|c|}{ Insatisfatório } & \multicolumn{3}{|c|}{ Regular } & \multicolumn{3}{|c|}{ Satisfatório } & \\
\hline & G1 (\%) & G2 (\%) & AT (\%) & G1 (\%) & G2 (\%) & AT (\%) & G1 (\%) & G2 (\%) & AT (\%) & \\
\hline \multicolumn{11}{|l|}{ Organização didático-pedagógica } \\
\hline 1. Articulação teoria-prática & 3,4 & 14,4 & 17,8 & 13,9 & 23,6 & 37,5 & 30,8 & 13,9 & 44,7 & .001 \\
\hline 2. Flexibilidade curricular & 4,3 & 17,8 & 22,1 & 20,2 & 18,8 & 38,9 & 23,6 & 15,4 & 38,9 & .001 \\
\hline 3. Interdisciplinaridade & 1,9 & 13,5 & 15,4 & 13,0 & 20,7 & 33,7 & 33,2 & 17,8 & 50,9 & .004 \\
\hline 4. Carga horária total curricular & 8,2 & 15,4 & 23,6 & 13,0 & 16,3 & 29,3 & 26,9 & 20,2 & 47,1 & .029 \\
\hline 5. Carga horária dos ambientes & 10,1 & 15,9 & 25,9 & 19,2 & 21,6 & 40,9 & 18,8 & 14,4 & 33,2 & .147 \\
\hline 6. Pertinência dos conteúdos & 12,5 & 22,1 & 34,6 & 16,3 & 22,1 & 38,4 & 19,2 & 7,7 & 26,9 & .001 \\
\hline 7. Bibliografia recomendada & 4,8 & 6,7 & 11,6 & 9,6 & 16,3 & 26,0 & 33,7 & 28,8 & 62,5 & .092 \\
\hline 8. Uso de metodologias ativas & 8,7 & 11,1 & 19,7 & 8,7 & 13,0 & 21,6 & 30,8 & 27,9 & 58,6 & .301 \\
\hline 9. Apoio psicológico & 36,5 & 40,4 & 77,0 & 8,7 & 8,7 & 17,3 & 1,9 & 1,9 & 5,8 & .955 \\
\hline 10. Apoio pedagógico & 23,1 & 27,9 & 50,9 & 15,4 & 15,9 & 31,3 & 9,6 & 8,2 & 17,8 & 639 \\
\hline 12. Relação entre o número de professores e de alunos & 23,6 & 24,0 & 47,6 & 15,9 & 14,9 & 30,8 & 8,7 & 13,0 & 21,6 & .457 \\
\hline 13. Integração com a comunidade & 1,9 & 9,6 & 11,6 & 8,7 & 11,1 & 19,7 & 37,5 & 31,3 & 68,8 & .002 \\
\hline 14. Atividades práticas (exceto internato) & 1,0 & 4,8 & 5,7 & 4,3 & 12,0 & 16,3 & 42,8 & 35,1 & 77,9 & .001 \\
\hline \multicolumn{11}{|l|}{ Corpo docente e tutorial } \\
\hline 15. Titulação & 3,8 & 8,7 & 12,5 & 8,2 & 13,5 & 21,6 & 36,1 & 29,8 & 65,9 & .024 \\
\hline 16. Atuação do colegiado & 11,1 & 21,2 & 32,2 & 17,3 & 19,7 & 37,0 & 19,7 & 11,1 & 30,8 & .003 \\
\hline 17. Relação com alunos (dentro e fora da sala de aula) & 2,4 & 3,8 & 6,2 & 12,0 & 12,5 & 24,5 & 33,7 & 35,6 & 69,2 & .773 \\
\hline \multicolumn{11}{|l|}{ Estrutura física } \\
\hline 17. Acessibilidade & 19,7 & 26,0 & 45,7 & 20,7 & 16,3 & 37,0 & 7,7 & 9,6 & 17,3 & .226 \\
\hline 18. Espaço em relação ao número de alunos & 11,5 & 11,1 & 22,6 & 9,6 & 13,0 & 22,6 & 26,9 & 27,9 & 54,8 & .673 \\
\hline 19. Equipamentos didáticos & 8,7 & 11,5 & 20,2 & 13,9 & 12,5 & 26,4 & 25,5 & 27,9 & 53,4 & .625 \\
\hline 20. Iluminação & 3,8 & 3,8 & 7,7 & 9,1 & 11,5 & 20,7 & 35,1 & 36,5 & 71,7 & .846 \\
\hline 21. Equipamentos de controle de temperatura & 4,3 & 2,9 & 7,2 & 8,2 & 10,6 & 18,8 & 35,6 & 38,5 & 74,0 & .557 \\
\hline 22. Tecnologias de informação & 20,2 & 22,1 & 42,4 & 13,0 & 12,0 & 25,0 & 14,9 & 17,8 & 32,7 & .786 \\
\hline 23. Quantidade de livros & 31,3 & 32,7 & 63,9 & 11,5 & 13,9 & 25,4 & 5,3 & 5,3 & 10,6 & .890 \\
\hline 24. Diversidade de títulos na biblioteca & 24,5 & 28,8 & 52,9 & 9,6 & 12,5 & 22,5 & 13,9 & 10,6 & 24,5 & .338 \\
\hline 25. Disponibilidade dos laboratórios em horário extra & 22,6 & 36,5 & 59,2 & 10,6 & 10,1 & 20,7 & 14,9 & 5,3 & 20,1 & .001 \\
\hline 26. Materiais e equipamentos laboratoriais & 15,9 & 19,7 & 35,6 & 16,3 & 18,3 & 34,6 & 15,9 & 13,9 & 29,8 & .595 \\
\hline 27. Áreas de estudo e convivência & 26,9 & 35,6 & 62,5 & 13,5 & 11,1 & 24,5 & 7,7 & 5,3 & 13,0 & .165 \\
\hline 28. Restaurante universitário & 7,2 & 4,3 & 11,6 & 11,1 & 9,1 & 20,2 & 29,8 & 38,5 & 68,3 & .145 \\
\hline \multicolumn{11}{|l|}{ Contribuição do currículo informal no aprendizado } \\
\hline 29. Centro Acadêmico & 10,6 & 18,8 & 29,3 & 17,8 & 17,8 & 35,6 & 19,7 & 15,4 & 35,1 & .062 \\
\hline 30. Associação Atlética Acadêmica & 8,7 & 15,4 & 24,0 & 16,3 & 14,4 & 30,8 & 23,1 & 22,1 & 45,2 & .142 \\
\hline 31. Ligas acadêmicas & 1,4 & 1,9 & 3,3 & 9,1 & 9,6 & 18,8 & 37,5 & 40,4 & 77,9 & .959 \\
\hline
\end{tabular}


ticas, titulação docente, relação docente e discente, adequação do espaço físico, equipamentos didáticos, iluminação, equipamentos de controle de temperatura, restaurante universitário e ligas acadêmicas. Por outro lado, foram avaliados como insuficientes o apoio psicológico e pedagógico ao discente, a relação entre o número de docentes e discentes, tecnologias de informação, quantidade e diversidade de livros na biblioteca, disponibilidade de laboratórios em horário extra, materiais e equipamentos laboratoriais, áreas de estudo e convivência, e acessibilidade.

Quanto à comparação entre os grupos, houve diferença estatística significativa com relação à articulação teoria-prática ( $\mathrm{p}=0,001)$, flexibilidade curricular ( $\mathrm{p}=0,001)$, interdisciplinaridade $(p=0,004)$, pertinência dos conteúdos $(p=0,001)$, integração com a comunidade $(\mathrm{p}=0,002)$, atividades práticas nos quatro anos iniciais ( $\mathrm{p}=0,001)$, atuação do colegiado ( $\mathrm{p}=$ 0,003 ) e disponibilidade dos laboratórios em horário extra ( $p$ $=0,001)$. Para todos os itens avaliados obteve-se maior nível da categoria insatisfatório para G2 e maior nível da condição satisfatório para G1.

A Tabela 3 apresenta a distribuição das frequências obtidas para questões da dimensão organização didático-pedagógica e sua relação com a contribuição das ligas acadêmicas no processo de aprendizagem do discente.

Não houve significância estatística na comparação entre a articulação teoria-prática $(p=0,226)$ e carga horária total curricular ( $p=0,6)$ com a contribuição das ligas acadêmicas. Entretanto, houve significância ao compararmos essa contribuição com a pertinência dos conteúdos $(\mathrm{p}=0,05)$ e a carga horária dos ambientes $(p=0,036)$.

\section{DISCUSSÃO}

A criação de um novo curso de graduação é sempre um processo árduo, que envolve mais do que simplesmente construir salas de aula. Isto se torna ainda mais visível no campo da Medicina, que envolve diversas especificidades, como a importância da integração dos ambientes acadêmicos com a comunidade, necessidade de formar profissionais crítico-reflexivos, capazes de compreender os anseios e necessidades da população, e maior tempo de formação. As dificuldades são ainda maiores ao compreendermos que a formação de novos profissionais na área da saúde passa por um processo de reestruturação, baseada cada vez mais nos princípios das metodologias ativas ${ }^{16,17}$. Neste contexto, é natural encontrar dificuldades durante o processo de implementação.

Identificamos que os alunos do segundo grupo (G2), que passaram pela mudança curricular, apontaram estar significativamente menos satisfeitos com a estrutura curricular e com a pertinência dos conteúdos curriculares. Isto revela uma dificuldade na transição e potencial prejuízo no aprendizado, uma vez que tiveram que se adaptar, em curto período de tempo, às novas estratégias de ensino e rotinas de estudo, sugerindo que um período maior de transição seria necessário ao considerarmos a forte influência que a metodologia tradicional teve na vida acadêmica destes discentes ${ }^{18}$. Apesar disso, não houve diferença estatística entre os grupos quanto à

\begin{tabular}{|c|c|c|c|c|c|c|c|c|}
\hline $\begin{array}{r}\text { Distribuição } \\
\text { sua relação }\end{array}$ & $\begin{array}{l}\text { equências ob } \\
\text { a contribuiçã }\end{array}$ & $\begin{array}{r}\mathrm{T} \\
\text { ues }\end{array}$ & $\begin{array}{l}3 \\
\text { a din no }\end{array}$ & $\begin{array}{l}\text { org } \\
\text { sso }\end{array}$ & $\begin{array}{l}\text { ção d } \\
\text { cendi }\end{array}$ & $\begin{array}{l}\text { co-p } \\
\text { n do }\end{array}$ & $\begin{array}{l}\text { gica e } \\
\text { ente }\end{array}$ & \\
\hline & & & buiçã & igas & iicas 1 & apre & & \\
\hline & & Ins & ório & & & & ório & $p$ \\
\hline & & $\mathrm{n}$ & $\%$ & $\mathrm{n}$ & $\%$ & $\mathrm{n}$ & $\%$ & \\
\hline & Insatisfatório & 3 & 1,4 & 10 & 4,8 & 24 & 11,5 & \\
\hline Articulação teoria-prática & Regular & 2 & 0,9 & 13 & 6,2 & 63 & 30,2 & .226 \\
\hline & Satisfatório & 2 & 0,9 & 16 & 7,7 & 75 & 36,1 & \\
\hline & Insatisfatório & 3 & 1,4 & 11 & 5,3 & 35 & 16,8 & \\
\hline Carga horária total curricular & Regular & 2 & 0,9 & 12 & 5,8 & 47 & 22,6 & .600 \\
\hline & Satisfatório & 2 & 0,9 & 16 & 7,7 & 80 & 38,5 & \\
\hline & Insatisfatório & 4 & 1,9 & 14 & 6,7 & 36 & 17,3 & \\
\hline Carga horária dos ambientes & Regular & 3 & 0,9 & 17 & 8,2 & 65 & 31,2 & .036 \\
\hline & Satisfatório & 0 & 0,0 & 8 & 3,8 & 61 & 29,3 & \\
\hline & Insatisfatório & 6 & 2,8 & 16 & 7,7 & 50 & 24,0 & \\
\hline Pertinência dos conteúdos & Regular & 21 & 10,1 & 11 & 5,3 & 48 & 23,1 & .050 \\
\hline & Satisfatório & 14 & 6,7 & 18 & 8,6 & 24 & 11,5 & \\
\hline
\end{tabular}


influência das metodologias ativas, estando ambos satisfeitos com este modelo, demonstrando sucesso em superar as limitações na mudança do papel do professor e a resistência dos próprios alunos ${ }^{19}$. Achados semelhantes são encontrados por diversos estudos realizados em instituições públicas da área da saúde em São Paulo ${ }^{20}$, Distrito Federal ${ }^{21}$ e Santa Catarina ${ }^{22}$, onde, além de se sentirem satisfeitos com a metodologia, os alunos identificaram outras vantagens, como valorização do trabalho em equipe e pensamento crítico-reflexivo.

A relação numérica entre alunos e professores foi considerada pelos discentes um fator desfavorável ao aprendizado, expondo a necessidade de reestruturação do corpo docente, com aumento do número de vagas, principalmente dentro das metodologias ativas, que se baseiam em grupos menores e relação mais direta com o docente ${ }^{19}$. Já as estruturas das salas de aula foram positivamente avaliadas em todos os aspectos analisados pelo estudo, fato esperado por se tratar de estruturas com pouco tempo de uso. Um estudo com alunos de uma instituição pública de ensino superior na Região Sul do Brasil ${ }^{23}$ aventou a possibilidade de estes considerarem que a infraestrutura não afeta diretamente a percepção da qualidade de ensino porque ao ingressarem no curso não tinham grandes expectativas com relação a este aspecto.

Também estatisticamente significativa foi a diferença entre os grupos quanto à insuficiência da integração com a comunidade e das atividades nos ambientes de práticas (Tabela 2), podendo estar relacionada com questões da própria limitação da rede de saúde, já que a universidade não possui hospital-escola próprio ou ambulatórios de diversas especialidades, estruturas geralmente relacionadas com os períodos intermediários, a partir do terceiro ano, e mais avançados, que, além da sua importância assistencial, são locais de ensino e pesquisa $^{24,25}$.

A disponibilidade do restaurante universitário foi avaliada positivamente e pode ser considerada relevante pela localização das instalações físicas do campus da universidade, possivelmente pela distância e consequente necessidade de permanecer no campus mesmo durante os intervalos entre as aulas. Por outro lado, os discentes apontaram falta de espaços de convivência e áreas de estudo, evidenciada pela porcentagem de insatisfação na Tabela 2 .

A biblioteca foi considerada insatisfatória quanto à diversidade de títulos à disposição e principalmente no tocante à quantidade, fatores contrários às políticas de ações afirmativas e inclusão crescente de estudantes em situação de vulnerabilidade econômica ${ }^{26}$, aumentando a dificuldade de formação profissional para os alunos que não têm condições de adquirir livros da área, geralmente de alto custo, fazendo com que te- nham que obter cópias ou livros digitalizados para seu apren$\operatorname{dizado}^{27}$

Tais limitações na infraestrutura são compreensíveis no contexto de um curso novo, em campus também novo, mas indubitavelmente resultam em dificuldades adicionais no processo de aprendizagem para os alunos das primeiras turmas.

Com relação aos apoios psicológico e pedagógico e à acessibilidade, tanto G1 quanto G2 concordaram em que são áreas insatisfatórias, especialmente a primeira. Trata-se de um importante ponto a ajustar, buscando-se analisar o perfil discente e encontrar estratégias para promoção de saúde mental ${ }^{28,29}$, considerando as dificuldades inerentes a qualquer graduação, onde muitos discentes são jovens e por vezes moram longe da família, ainda mais ao considerarmos a elevada carga emocional e de estudos que envolve a área médica ${ }^{30,31}$. Tais achados são compatíveis com os encontrados em estudo realizado no Ceará $^{32}$, que, além de apontar maior prevalência de distúrbios psicológicos nos discentes em comparação à população geral, também destacou a dificuldade dos futuros médicos de encontrar serviços de apoio especializados em suas instituições formadoras.

Nesta perspectiva, ressaltamos a importância, apontada pelos discentes, das atividades complementares e do currículo informal, caso das atividades em ligas acadêmicas, Centro Acadêmico e Associação Atlética Acadêmica, em especial esta última. Isto porque, mesmo nos semestres mais avançados, em que os alunos tendem a direcionar suas atividades às práticas médicas, observamos ainda satisfação discente, possivelmente relacionada à necessidade da prática desportiva como forma de diminuir o estresse ${ }^{33}$

Um aspecto bastante revelador foi evidenciado ao compararmos a contribuição das ligas acadêmicas com as questões curriculares (Tabela 3), onde a significância estatística na comparação com a pertinência dos conteúdos $(\mathrm{p}=0,05)$ pode significar que, apesar das dificuldades de implantação do novo currículo, a estruturação está adequada, mas os assuntos não estão sendo suficientes para os acadêmicos, que buscam alternativas para complementar sua formação médica e ampliar sua bagagem científica ${ }^{34,35}$. Torna-se importante discutirmos, no entanto, os riscos de tamanha atribuição de atividades informais em contraposição ao currículo formal, tendo em vista que este possui diversos vieses, inclusive de subversão curricular e promoção de vícios acadêmicos. É, portanto, prioritário replanejar o currículo atual para que os estudantes não sintam a necessidade de buscar em atividades extras o que acreditam ser fundamental para sua formação acadêmica ${ }^{36}$

Cabe ainda ressaltar que todas as mudanças e ajustes no projeto pedagógico do curso (PPC) foram mediados por pro- 
dutivas discussões entre os corpos docente e discente, que incluíam desde ajustes nos conteúdos e bibliografias utilizadas, até a carga horária disponibilizada para cada tema, em esforço coletivo para a construção do ambiente mais adequado ao aprendizado, demonstrando a natureza participativa dos discentes nas metodologias ativas.

\section{CONCLUSÃO}

Podemos verificar, com base nos aspectos avaliados e nos resultados obtidos, que a maioria dos discentes é favorável ou está satisfeita, demonstrando que, apesar das dificuldades na transição da metodologia ativa, especialmente nesse curso de graduação relativamente novo, existem vários pontos positivos que podem ser adequados para seu aprendizado e formação acadêmica, incluindo a boa relação com os docentes, salas de aula com estrutura adequada e contribuição do currículo informal, em especial das ligas acadêmicas e Associação Atlética Acadêmica.

Entretanto, existem ainda pontos-chave que necessitam reestruturações, como contratação de mais docentes, melhor suporte psicopedagógico, com desenvolvimento de novas estratégias de acompanhamento discente, aquisição em quantidade e diversidade de títulos para a biblioteca e melhoria da acessibilidade, a fim de facilitar o processo de aprendizagem dos estudantes.

\section{REFERÊNCIAS}

1. D'Costa MP, Swarnadas GS. Students' perceptions of effective clinical teaching and teacher behaviours on learning. Manipal Journal of Nursing and Health Sciences. 2016;2(2):1-8

2. Ismail LM, Aboushady RM, Eswi A. Clinical instructor's behavior: nursing student's perception toward effective clinical instructor characteristics. Journal of Nursing Education and Practice. 2016;6(2):96-105.

3. Siqueira DCT. Relação professor-aluno: uma revisão crítica. Integração. 2003;33(1):97-101.

4. Carroll RG. It's all about the teacher-student relationship. Advances in Physiology Education. 2012;36(1):233.

5. Mitre SM, Siqueira-Batista R, Girardi-de-Mendonça JM, Morais-Ponto NM, Meirelles CAB, Pinto-Porto C et al. Metodologias ativas de ensino-aprendizagem na formação profissional em saúde: debates atuais. Ciência \& Saúde Coletiva. 2008;13(2):2133-44.

6. Conselho Nacional de Educação Câmara de Educação Superior. Resolução CNE/CES 4/2001 (2001).

7. Conselho Nacional de Educação Câmara de Educação Superior. Resolução CNE/CES 3/2014 (2014).
8. Bartolo EB, dos Santos MAP, Dinato MC, Pinto RMF. Humanidades Médicas - Metodologia Utilizada no Curso de Medicina do Centro Universitário Lusíada (Unilus). Revista Brasileira de Educação Médica. 2017;41(3):449-53.

9. Santos LMP, Costa AM, Girardi SN. Programa Mais Médicos: uma ação efetiva para reduzir iniquidades em saúde. Ciência \& Saúde Coletiva. 2015;20(11):3547-52.

10. Schleich ALR, Polydoro SAJ, dos Santos AAA. Escala de satisfação com a experiência acadêmica de estudantes do ensino superior. Avaliação Psicológica. 2006;5(1):11-20.

11. Instituto Brasileiro de Geografia e Estatística. Diretoria de Pesquisas, Coordenação de População e Indicadores Sociais (2018).

12. Departamento de Informática do Sistema Único de Saúde. Informações de saúde: TABNET (2018).

13. Henzi D, Davis E, Jasinevicius R, Hendricson W, Cintron L, Isaacs M. Appraisal of the dental school learning environment: the students' view. Journal of Dental Education. 2005;69(10):1137-47.

14. Braccialli LAD, Oliveira MAC. Desafios na Formação Médica: a Contribuição da Avaliação. Revista Brasileira de Educação Médica. 2012;36(2):280-88.

15. Instituto Nacional de Estudos e Pesquisas Educacionais Anísio Teixeira. Instrumento de Avaliação de Cursos de Graduação - presencial e a distância (2016).

16. Gomes MPC, Ribeiro VMB, Monteiro DM, Leher EMT, Louzada RCR. O uso de metodologias ativas no ensino de graduação nas ciências sociais e da saúde - avaliação dos estudantes. Ciência e Educação. 2010;16(1):181-198.

17. Almeida EG, Batista NA. Desempenho docente no contexto PBL: essência para aprendizagem e formação médica. Revista Brasileira de Educação Médica. 2012;37(2):192-201.

18. Cezar PHN, Guimarães FT, Gomes AP, Rôças G, Siqueira-Batista R. Transição Paradigmática na Educação Médica: Um Olhar Construtivista Dirigido à Aprendizagem Baseada em Problemas. Revista Brasileira de Educação Médica. 2010;34(2):298-303.

19. Borges MC, Chachá SGF, Quintana SM, Freitas LCC, Rodrigues MLV. Aprendizado baseado em problemas. Medicina (Ribeirão Preto). 2014;47(3):301-7.

20. Paranhos VD, Mendes MMR. Currículo por competência e metodologia ativa: percepção de estudantes de enfermagem. Ver Latino-Am. de Enfermagem. 2010;18(1):1-7.

21. Melo BC, Sant'Ana G. A prática da metodologia ativa: compreensão dos discentes enquanto autores do processo ensino-aprendizagem. Com Ciências Saúde. 2012;23(4):327-39.

22. Prado ML, Velho MB, Espíndola DM, Sobrinho SH, Backes VMS. Arco de Charles Maguerez: refletindo estratégias de 
metodologia ativa na formação de profissionais de saúde. Esc Anna Nery. 2012;16(1):172-7.

23. Walter S, Neto P, Prado P, Tontini G. Percepção da qualidade de ensino em uma instituição pública de ensino superior. Um estudo multimétodos. Rev Portuguesa e Brasileira de Gestão. 2011;10(3):48-59.

24. Feuerwerker LCM, Cecílio LCO. O hospital e a formação em saúde: desafios atuais. Ciência e Saúde Coletiva. 2007;12(4):965-71.

25. Araújo KM, Leta J. Os hospitais universitários federais e suas missões institucionais no passado e no presente. 2014;21(4):1261-81.

26. Oliveira AJB, Cranchi DC. O papel da biblioteca universitária como espaço de afiliação estudantil e o bibliotecário como educador e agente inclusivo. Informação e Sociedade: Estudos. 2017;27(2):35-47.

27. Albuquerque FC, Paiva EB. Livros digitalizados: uso e satisfação de usuários da UFPB. Biblionline. 2016;11(1)35-57.

28. Pereira AMS, Motta ED, Vaz AL, Pinto C, Bernardino O, Melo AC et al. Sucesso e desenvolvimento psicológico no Ensino Superior: Estratégias de intervenção. Análise psicológica. 2006;24(1):51-9.

29. Bisinoto C, Marinho-Araújo C. Sucesso acadêmico na Educação Superior: Contribuições da Psicologia Escolar. Revista E-Psi. 2014;4(1):28-46.

30. Silva GCC, Sousa EG, Martins LAN, Buys RC, Santos AASMD, Koch HA. A importância do apoio psicológico ao médico residente e especializando em radiologia e diagnóstico por imagem. Radiol Bras. 2011;44(2):81-4.

31. Côrtes PPR, Vilagra SMBW, Souza MCA, Côrtes Júnior JCS, Rabello E. Estratégias para a ressignificação do internato médico: relato de experiência. Revista Ciência e Estudos Acadêmicos de Medicina. 2016;1(5):42-52.

32. Andrade JBC, Sampaio JJC, Farias LM, Melo LP, Sousa DP, Mendonça ALB et al. Contexto de Formação e Sofrimento Psíquico de Estudantes de Medicina. Revista Brasileira de Educação Médica. 2014;38(2):231-42.
33. Peres CM, Andrade AS, Garcia SB. Atividades Extracurriculares: Multiplicidade e Diferenciação Necessárias ao Currículo. Revista Brasileira de Educação Médica. 2007;31(2):147-155.

34. Botelho NM, Ferreira IG, Souza LEA. Ligas acadêmicas de medicina: artigo de revisão. Rev. Paraense Med. 2013;27(4):85-8.

35. Queiroz SJ, Azevedo RLO, Lima KP, Lemes MMD, Andrade M. A importância das Ligas Acadêmicas na formação profissional e promoção de saúde. Fragmentos de cultura. 2014;24(1):73-8.

36. Hamamoto Filho PT. Ligas Acadêmicas: Motivações e Críticas a Propósito de um Repensar Necessário. Revista Brasileira de Educação Médica. 2011;35(4):535-43.

\section{CONTRIBUIÇÃO DOS AUTORES}

Ambos os autores foram responsáveis e contribuíram para a concepção e desenho do estudo, aquisição, análise e interpretação dos dados. Também contribuíram para a elaboração do artigo e revisão crítica do conteúdo, sendo igualmente responsáveis pela versão final publicada.

\section{CONFLITO DE INTERESSES}

Os autores declaram não haver conflito de interesses. O financiamento da pesquisa foi realizado pelos autores.

\section{ENDEREÇO PARA CORRESPONDÊNCIA}

Paulo Roberto Sotillo de Lima Filho

Centro de Ciências Sociais, Saúde e Tecnologia - Campus Avançado - UFMA

Av. da Universidade, S/N

CEP: 65915-240

Imperatriz - MA

E-mail: paulosotillo@gmail.com 